\title{
Predação de Drymoluber Dichrous (Reptilia: Squamata: Colubridae) por Acanthoscurria sp. (Araneae: Theraphosidae) no Sudoeste da Amazônia Brasileira
}

Serpentes não são componentes usuais na dieta de invertebrados, tornando o hábito de ofiofagia por tarântulas incomum na natureza. Existem poucos estudos sobre a biologia das serpentes do sudoeste da Amazônia quando comparadas com as partes centrais e orientais deste mesmo bioma. Pesquisas sobre dietas de vertebrados podem ser realizadas, além das observações de eventos de predação, com a dissecação do conteúdo, porém invertebrados como as tarântulas não retêm partes do corpo das presas durante a digestão e consomem frequentemente apenas material corporal liquefeito. É relatado no presente trabalho uma observação de predação por uma aranha Acanthoscurria sp. sobre uma serpente Drymoluber dichrous em um fragmento de floresta na área rural da cidade de Porto Velho, no sul da Amazônia brasileira, estado de Rondônia. Este relato é considerado um evento raro pois aponta um hábito alimentar incomum para aranhas do gênero Acanthoscurria. Durante uma pesquisa de campo, os autores desta comunicação se depararam com este registro. Acreditava-se que a serpente estava tentando se abrigar, e quando removida, constatou-se que estava em processo de digestão extracorpórea pela aranha. Acredita-se que acidentalmente, a serpente de pequeno porte tentou se abrigar na toca habitada pela aranha e foi surpreendida. Registros deste tipo expandem o conhecimento sobre os hábitos alimentares das aranhas do gênero Acanthoscurria e são importantes para aumentar o conhecimento sobre os predadores das serpentes da espécie Drymoluber dichrous.

\section{Predation of Drymoluber Dichrous (Squamata: Colubridae) by Acanthoscurria sp. (Araneae: Theraphosidae) in Southern Brazilian Amazon}

Snakes are not common components in the invertebrate's diet, making the habit of tarantula ophiophagy uncommon in nature. There are few studies on the biology of snakes in the southwestern Amazon when compared to the central and eastern parts of this same biome. Research on vertebrate diets can be carried out, besides the observations of predation events, with the dissection of the content, but invertebrates such as tarantulas do not retain body parts of the prey during digestion and frequently consume only liquefied body material. We reported in this work a predation observation by a spider Acanthoscurria sp. on a Drymoluber dichrous snake in a forest fragment in a rural area of the city of Porto Velho, in the southern Brazilian Amazon, state of Rondônia. This account is considered a rare event as it points out an unusual feeding habit for spiders of the genus Acanthoscurria. During field research, the authors of this paper came across this record. It was believed that the snake was trying to shelter itself, and when removed, it was found to be in the process of extracorporeal digestion by the spider. It is believed that the small snake accidentally tried to shelter in the burrow inhabited by the spider and was surprised. Records of this kind expand the knowledge about the feeding habits of spiders of the genus Acanthoscurria and are important to increase the knowledge about the predators of the Drymoluber dichrous species.

Keywords: Rondônia; Spider; Ophiophagy; Prey; Amazon rainforest.

Topic: Notas Científicas

Reviewed anonymously in the process of blind peer
Received: 06/03/2021

Approved: 27/05/2021
Raul Afonso Pommer Barbosa

Fundação Getúlio Vargas, Brasil

http://lattes.cnpq.br/9136549262197723

http://orcid.org/0000-0002-1914-5184

raulpommer@hotmail.com

Isabella Karen Assis da Silva

Centro Universitário São Lucas, Brasil

http://lattes.cnpq.br/6196130972916967

isabellakaren@hotmail.com

Uéslei Marques de Oliveira

Centro Universitário São Lucas, Brasil

http://lattes.cnpq.br/3034343125833374

uesleimarques@hotmail.com

○

DOI: 10.6008/CBPC2318-2881.2021.002.0019
Saymon de Albuquerque (D)

Centro Universitário São Lucas, Brasil

http://lattes.cnpq.br/7130569683759838

http://orcid.org/0000-0002-5022-6724

saymon albuquerque@hotmail.com

Referencing this:

BARBOSA, R. A. P.; SILVA, I. K. A.; OLIVEIRA, U. M.; ALBUQUERQUE, S. Predação de Drymoluber Dichrous (Reptilia: Squamata: Colubridae) por Acanthoscurria sp. (Araneae: Theraphosidae) no Sudoeste da Amazônia Brasileira. Nature and Conservation, v.14, n.2, p.213-218, 2021. DOI: http://doi.org/10.6008/CBPC2318-2881.2021.002.0019 


\section{INTRODUÇÃO}

Serpentes não são componentes usuais na dieta de invertebrados, tornando o hábito de ofiofagia por tarântulas incomum na natureza (BORGES et al., 2016). Existem poucos estudos sobre a biologia das serpentes do sudoeste da Amazônia quando comparadas com as partes centrais e orientais deste mesmo bioma (MARTINS et al., 1998; CUNHA et al., 1993).

Pesquisas sobre dietas de vertebrados podem ser realizadas, além das observações de eventos de predação, com a dissecação do conteúdo estomacal (BERNARDE et al., 2006; BERNARDE et al., 2010; COSTA et al., 2013), porém invertebrados como as tarântulas não retêm partes do corpo das presas durante a digestão e consomem frequentemente apenas material corporal liquefeito (FOELIX, 2011; NORDBERG et al., 2018). Por realizar a digestão extracorpórea, os estudos sobre os hábitos alimentares das tarântulas ficam limitados a observações de eventos de predação, indicando a importância de registros deste tipo. Aqui, registramos a predação da Drymoluber dichrous (Peters, 1863) por Acanthoscurria sp. (Ausserer, 1871) no sul da Amazônia brasileira.

The northern woodland racer, Drymoluber dichrous (Peters, 1863) é uma serpente Colubridae distribuída na região do escudo da Guiana (ÁVILA-PIRES, 2005; COLE et al., 2013). Também é encontra na Colômbia, Equador, Peru oriental, norte do Brasil, Venezuela amazónica (PETERS et al., 1970; NASCIMENTO et al., 1988; COLE et al., 2013). Em Rondônia é encontrada nos municípios de Porto Velho, Guajará-Mirim, Cacoal e Espigão do Oeste (BERNARDE et al., 2006; BERNARDE et al., 2012), distribuída em áreas de floresta, floresta terra-firme, vegetação secundaria, pastos e áreas com um relativo grau de antropização (CUNHA et al., 1978; MARTINS et al., 1998; BERNARDE et al., 2006; BERNARDE et al., 2010).

Encontrada com muita frequência (BERNARDE et al., 2012), é descrita como uma serpente com hábitos diurnos e terrestres, que ao anoitecer descansa sobre as folhas da vegetação (BERNARDE et al., 2006), como uma forma de evitar ser vítima de predadores terrestres (MARTINS, 1993). Sua dieta é composta por anuros, lagartos (BERNARDE et al., 2006; BERNARDE et al., 2010), ovos de lagartos, serpentes (Oxybelis, Drymoluber) (MARTINS et al., 1998; PINTO, 2006), filhotes de gambás (Didelphidae) e Anfisbenas (COSTA et al., 2013). Sobre os mecanismos de defesa, cabe dizer que um espécime de Porto Velho, Rondônia, foi descrito como muito agressivo, atacando e vibrando frequentemente a cauda (O.A.V. MARQUES, pers. comm 1997; MARTINS et al., 1998). Existem evidências do comportamento defensivo conhecido como pseudoautotomia (COSTA et al., 2014). Os predadores conhecidos desta espécie são aves, como gaviões e corujas, e outras serpentes (SAWAYA et al., 2008; COSTA et al., 2014).

Acanthoscurria é um gênero de tarântulas que foi descrito pela primeira vez por Anton Ausserer, em 1871 e contém 27 espécies descritas (WSC, 2020) distribuídas na América do Sul, nas Antilhas e a Guatemala. No Brasil, este gênero está distribuído nos estados de Rondônia, Roraima, Amazonas, Acre, Pará, Mato Grosso do sul e Mato Grosso (PAULA et al., 2014). As espécies amazônicas são revistas e redescritas com base no tipo de material e em espécimes da coleção do Instituto Butantan e outras importantes coleções aracnológicas brasileiras (PAULA et al., 2014). As espécies que ocorrem em Rondônia são: Acanthoscurria 
Predação de Drymoluber Dichrous (Reptilia: Squamata: Colubridae) por Acanthoscurria sp. (Araneae: Theraphosidae) no Sudoeste da Amazônia Brasileira

BARBOSA, R. A. P.; SILVA, I. K. A.; OLIVEIRA, U. M.; ALBUQUERQUE, S.

geniculata (Koch, 1841), Acanthoscurria juruenicola (Mello-Leitão, 1923); Acanthoscurria theraphosoides (Doleschall, 1871); Acanthoscurria insubtilis (Simon, 1892).

De maneira geral, a Acanthoscurria ssp. do estado de Rondônia possuem hábito noturno, mas também podem ser encontradas durante o dia. Cavam tocas tabulares sob rochas, troncos caídos em barrancos ou no chão. Além disso, outras Theraphosidae e grande parte dos migalomorfos possuem estes hábitos (INDICATTI et al., 2008; COYLE, 1971; LUCAS et al., 2005; 2008; PAULA et al., 2014), sendo encontradas em zonas de floresta, estradas e matas urbanas. Algumas espécies do gênero Acanthoscurria possuem comportamento defensivo agressivo, podendo levantar as patas anteriores e mostrar as quelíceras, e em alguns casos, liberando algumas gotas de veneno (PAULA et al., 2014; PÉREZ-MILES et al., 2017). As aranhas produzem veneno que utilizam para apanhar as presas e iniciar o processo de digestão. Como não possuem dentes, devem liquefazer as suas vítimas antes de poderem ingerir (SAUCIER, 2004). As picadas de tarântulas (Theraphosidae, Mygalomorphae) são consideradas dolorosas, mas não induzem necrose local ou efeitos sistémicos em humanos (SAUCIER, 2004; MOURÃO, 2013). O hábito alimentar é descrito em uma dieta que consiste em pequenos artrópodes, como besouros e baratas (PÉREZ-MILES et al., 2017). Existem alguns registros que descrevem a predação de Theraphosidae em répteis e anfíbios (VIEIRA et al., 2012; BORGES et al., 2016; PINTO et al., 2017).

\section{RELATO}

A predação foi observada durante uma ida a campo no Centro de Cultura e Formação Kanindé ( $\left.8^{\circ} 53^{\prime} 58.4^{\prime \prime} \mathrm{S} 63^{\circ} 53^{\prime} 48.9^{\prime \prime} \mathrm{W}\right)$ no município de Porto Velho, Rondônia. O local fica na zona rural, seguindo pela Estrada da Areia Branca. É um fragmento de floresta tropical amazônica com aproximadamente $2 \mathrm{~km}^{2}$ de dimensão. 0 ponto exato da predação fica a 33,6 metros de um lago artificial.; 144,50 metros da Estrada da Areia Branca e 81,15 metros de uma zona desmatada, indicando assim a existência de um impacto humano relativo no local.

Os pesquisadores estavam realizando um levantamento de herpetofauna com busca ativa e fotografia dos animais encontrados. No dia 15 de junho (sexta-feira) de 2018, às 22:47:48 foi observado que havia uma pequena serpente em um tronco, pois a parte posterior dela estava exposta. Ao tentar capturar a serpente, foi constatado que ela estava sendo predada por uma tarântula Acanthoscurria sp. e que parte dela estava em processo de digestão extracorpórea (Figura 1A e 1C). $\mathrm{O}$ processo de digestão e a lesão encontrada são similares com o de estudos anteriores (BORGES et al., 2016). Procedeu-se com fotografias para futura identificação com câmera fotográfica Nikon P900 e anotações nos cadernos de campo. Houve a tentativa de se retirar a aranha de sua toca, sem sucesso, porém sendo registradas fotos de suas partes expostas (Figura 1B). 


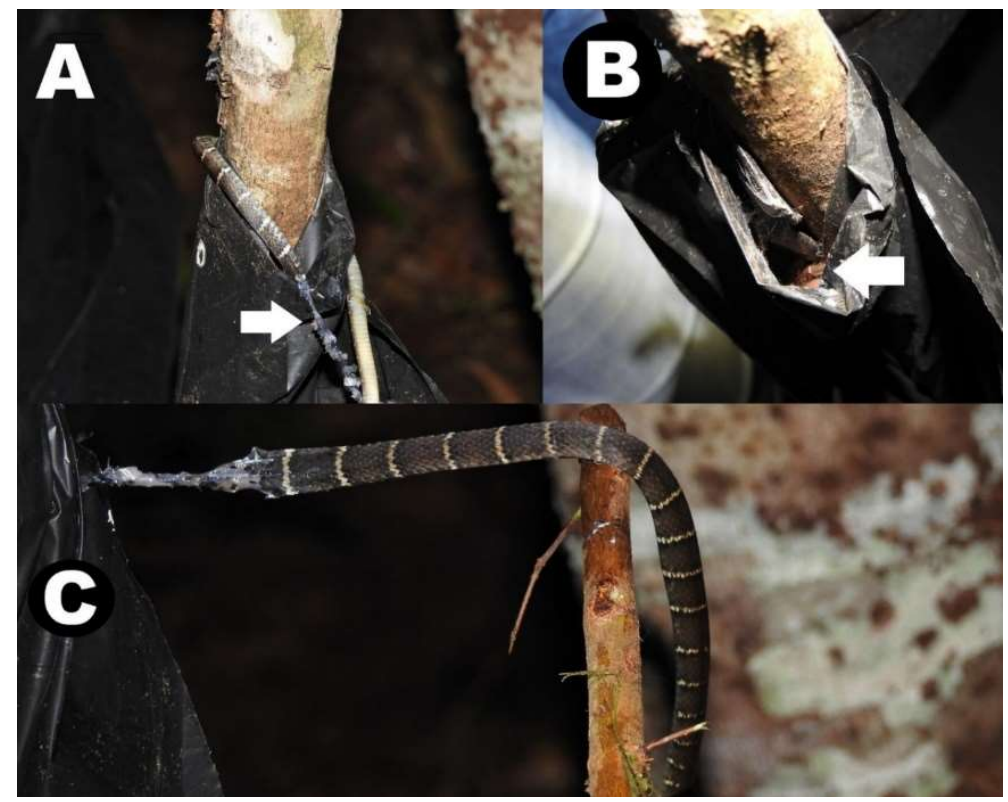

Figura 1: Evidência fotográfica da Predação da Drymoluber dichrous (Reptilia: Squamata: Colubridae) por Acanthoscurria sp. (Araneae: Theraphosidae). A: Lesão da digestão extracorpórea. B: Toca da Acanthoscurria sp.; C: Restos mortais não digeridos da Drymoluber dichrous.

\section{DISCUSSÃO}

A serpente foi identificada como sendo Drymoluber dichrous juvenil, por apresentar o dorso pardo e faixas circulares estreitas na cor amarelo-creme (MARTINS et al., 1998; MARÇAL et al., 2011; COSTA et al., 2013) e a tarântula Acanthoscurria sp. (PAULA et al., 2014; PÉREZ-MILES et al., 2017). As medidas anotadas no caderno de campo descrevem que a tarântula tinha por volta de $8 \mathrm{~cm}$ de tamanho. A serpente em digestão extracorpórea com $40 \mathrm{~cm}$ de comprimento e estimado em $60 \mathrm{~cm}$ totais. Acredita-se que acidentalmente, a serpente tentou se abrigar na toca habitada pela aranha e foi surpreendida, corroborando com o relato de Borges et al. (2016).

Apesar de diversos pesquisadores relatarem eventos de predação de aranhas em hepertofauna de regiões neotropicais (BARBO et al., 2009; MAFFEl et al., 2010; BOCCHIGLIERI et al., 2010; MOURA et al., 2011; DINIZ, 2011; BORGES et al., 2016), há bem menos exemplos de invertebrados documentados como predadores de vertebrados (NYFFELER et al., 2013; NYFFELER et al., 2017; NORDBERG et al., 2018). Os registros dos predadores da serpente Drymoluber dichrous são escassos e não há descrições mais detalhadas dos eventos predatórios (VERÍSSIMO et al., 2012).

\section{CONSIDERAÇÕES FINAIS}

Poucos eventos de predação de serpentes por tarântulas foram relatados na região amazônica. Registros deste tipo expandem o conhecimento sobre os predadores das serpentes da espécie Drymoluber dichrous e sobre os hábitos alimentares das aranhas do gênero Acanthoscurria ssp. indicando que este comportamento pode ser comum. É necessário que pesquisas futuras relatem e busquem entender a relação de presa-predador entre diferentes animais. 
Predação de Drymoluber Dichrous (Reptilia: Squamata: Colubridae) por Acanthoscurria sp. (Araneae: Theraphosidae) no Sudoeste da Amazônia Brasileira

\section{REFERÊNCIAS}

AUSSERER, A.. Beiträge zur Kenntniss der ArachnidenFamilie der Territelariae Thorell (Mygalidae Autor). Verhandlungen der Kaiserlich-Königlichen ZoologischBotanischen Gesellschaft in Wien, 1871.

ÁVILA-PIRES, T. C. S.. Reptiles. In: HOLLOWELL, T.; REYNOLDS, R. P.. Checklist of the Terrestrial Vertebrates of the Guiana Shield. Bulletin of the Biological Society of Washington, v.13, p.1-98, 2005. p.25-40.

BARBO, F. E.; RODRIGUES, M. G.; COUTO, F. M.; SAWAYA, R. J.. Predation on Leptodactylus marmoratus (Anura: Leptodactylidae) by the spider Ctenus medius (Araneae: Ctenidae) in the Atlantic Forest, southeast Brazil. Herpetol. Notes., v.2, p.99-100, 2009.

BERNARDE, P. S.; ABE, A. S.. A Snake Community at Espigão Do Oeste, Rondônia, Southwestern Amazon, Brazil. South American Journal of Herpetology, v.1, n.2, p.102-113, 2006. DOI: https://doi.org/10.2994/18089798(2006)1[102:ascaed]2.0.co;2

BERNARDE, P. S.; ABE, A. S.. Hábitos alimentares de serpentes em Espigão do Oeste, Rondônia, Brasil. Biota Neotropica, v.10, n.1, p.167-173, 2010. DOI: https://doi.org/10.1590/s1676-06032010000100017

BERNARDE, P. S.; ALBUQUERQUE, S.; BARROS, T. O.; TURCI, L. C. B.. Serpentes do Estado de Rondônia, Brasil. Biota Neotrop., v.12, n.3, 2012.

BOCCHIGLIERI, A.; MENDONÇA, A. F.. Tropidurus oreadicus (neotropical ground lizard). Predation. Herpetol. Rev., v.41, p.231-231, 2010.

BORGES, L. M.; ROSA, C. M.; DRI, G. F.; BERTANI, R.. Predation of the snake Erythrolamprus almadensis (Wagler, 1824) by the tarantula Grammostola quirogai Montes De Oca, D’Elía \& Pérez-Miles. Herpetology Notes, v.9, p.321322, 2016.

COLE, C. J.; TOWNSEND, C. R.; REYNOLDS, R. P.; MACCULLOCH, R. D.; LATHROP, A.. Amphibians and reptiles of Guyana, South America: illustrated keys, annotated species accounts, and a biogeographic synopsis. Proceedings of the Biological Society of Washington, v.125, n.4, p.317578, 2013. DOI: https://doi.org/10.2988/0006-324x$\underline{125.4 .317}$

COSTA, H. C.; MOURA, M. R.; FEIO, R. N.. A tale of lost tails: pseudoautotomy in the Neotropical snake genus Drymoluber (Serpentes: Colubridae). Canadian Journal of Zoology, v.92, n.9, p.811-816, 2014. DOI: https://doi.org/10.1139/cjz-2014-0115

COSTA, H. C.; MOURA, M. R.; FEIO, R. N.. Taxonomic revision of Drymoluber Amaral, 1930 (Serpentes: Colubridae). Zootaxa, v.3716, n.3, p.349, 2013. DOI:

https://doi.org/10.11646/zootaxa.3716.3.3

COYLE, F.. Systematics and natural history of the mygalomorph genus Antrodiaetus and related genera (Araneae: Antrodiaetidae). Bulletin of the Museum of Comparative Zoology, v.141, p.269-402, 1971.
CUNHA, O. R.; NASCIMENTO, F. P.. Ofídios da Amazônia X: As cobras da região leste do Pará. Publicações Avulsas do Museu Paraense Emílio Goeldi, v.31, p.1-218, 1978.

CUNHA, O. R.; NASCIMENTO, F. P.. Ofídios da Amazônia. As cobras da região Leste do Pará. Boletim do Museu Paraense Emílio Goeldi série Zoologia, v.9, p.1-191, 1993.

DINIZ, S.. Predation and feeding on the tropical house gecko Hemidactylus mabouia (Squamata: Gekkonidae) by the giant orbweaver spider Nephilengys cruentata (Araneae: Nephilidae). Herpetol. Notes, v.4, p.357-358, 2011.

FOELIX, R.. Biology of Spiders. New York: Oxford University Press, 2011.

INDICATTI, R. P.; LUCAS, S. M.; GUADANUCCI, J. P. L.; YAMAMOTO, F. U.. Revalidation and revision of the genus Magulla Simon, 1892 (Araneae, Mygalomorphae, Theraphosidae). Zootaxa, v.1814, p.21-36, 2008.

$\mathrm{KOCH}, \mathrm{C}$. L.. Die Arachniden. C. H. Zeh'sche Buchhandlung, Nürnberg, Achter Band, pp. 41-131, pl. 265-288 (f. 621-694); Neunter Band, p.1-56, pl.289-306 (f. 695-726), 1841. DOI: https://doi.org/10.5962/bhl.title.43744

LUCAS, S. M.; INDICATTI, R. P.; FUKAMI, C. Y.. Redescrição de Prorachias bristowei Mello-Leitão 1924 (Araneae, Mygalomorphae, Nemesiidae). Biota Neotropica, v.5, n.1a, p.1-6, 2005. DOI: https://doi.org/10.1590/S1676$\underline{06032005000200019}$

LUCAS, S. M.; PASSANHA, V.; JANINI, C. R. V.; INDICATTI, R. $P$.. On the genus Neostothis Vellard (Araneae, Nemesiidae). Journal of Arachnology, v.36, p.472-475. 2008. DOI: https://doi.org/10.1636/CA07-107.1

MAFFEI, F.; UBAID, F. K.; JIM, J.. Predation of herps by spiders (Araneae) in the Brazilian Cerrado. Herpetol. Notes, v.3, p.167-170, 2010.

MARÇAL, A. S.; GOMES, I. B. R.; CORAGEM, J. T.. UHE Santo Antônio: Guia das espécies de fauna resgatadas. Porto Velho: Scriba Comunicação Corporativa, 2011.

MARTINS, M.; OLIVEIRA, M. E.. Natural history of snakes in forests of the Manaus region, Central Amazonia, Brazil. Herpetological Natural History, v.6, p.78-150, 1998.

MARTINS, M.. Why do snakes sleep on the vegetation in Central Amazonia?. Herpetological Review, v.24, n.83-84, p.1993.

MELLO-LEITÃO, C. F.. Theraphosideas do Brasil. Revista do Museu Paulista, v.13, p.1-438, 1923.

MOURA, M. R.; AZEVEDO, L. P.. Observation of predation of the giant fishing spider Ancylometes rufus (Walckenaer, 1837) (Araneae, Ctenidae) on Dendropsophus melanargyreus Cope, 1877 (Anura, Hylidae). Biota Neotropica, v.11, n.4, 2011. DOI: http://dx.doi.org/10.1590/S1676-06032011000400028 
MOURÃO, C. B. F.; OLIVEIRA, F. N.; CARVALHO, A. C.; ARENAS, C. J.; DUQUE, H. M.; GONÇALVES, J. C.; SCHWARTZ, E. F.. Venomic and pharmacological activity of Acanthoscurria paulensis (Theraphosidae) spider venom. Toxicon, v.61, p.129-138, 2013. DOI: https://doi.org/10.1016/j.toxicon.2012.11.008

NASCIMENTO, F. P.; ÁVILA-PIRES, T. C. S.; CUNHA, O. R.. Répteis Squamata de Rondônia e Mato Grosso coletados através do programa Polonoroeste. Bol. Mus. Para. Emílio Goeldi, Sér. Zool., v.4, p.21-66, 1988.

NORDBERG, E. J.; EDWARDS, L.; SCHWARZKOPF, L.. Terrestrial invertebrates: An underestimated predator guild for small vertebrate groups. Food Webs, v.15, e00080, 2018 DOI: https://doi.org/10.1016/j.fooweb.2018.e00080

NYFFELER, M.; MAXWELL, M. R.; REMSEN, J. V.. Bird predation by praying mantises: a global perspective. Wilson J. Ornithol., v.129, p.331-344, 2017. DOI: https://doi.org/10.1676/16-100.1

NYFFELER, M.; KNORNSCHILD, M.. Bat predation by spiders. PLoS ONE, v.8, e58120, 2013. DOI:

https://doi.org/10.1371/journal.pone.0058120

PAULA, F.S.; GABRIEL, R.; INDICATTI, R. P.; BRESCOVIT, A. D.; LUCAS, S. M.. On the Brazilian Amazonian species of Acanthoscurria (Araneae: Theraphosidae). Zoologia, Curitiba, v.31, n.1, p.63-80, 2014. DOI: https://doi.org/10.1590/S1984-46702014000100008

PÉREZ-MILES, F.; PERAFÁN, C.. Behavior and Biology of Mygalomorphae. Behaviour and Ecology of Spiders, 29-54. 2017. DOI: https://doi.org/10.1007/978-3-319-65717-2 2

PETERS, J. A.; OREJAS-MIRANDA, B.. Catalogue of the neotropical Squamata: Part I. Snakes. United States National Museum Bulletin 297. Washington: Smithsonian Institution
Press, 1970.

PINTO, K. C.; WRONSKI, L.; XAVIER, I.; PENHACEK, M.; OLIVEIRA, R. M.; OLIVEIRA, E. A.. Oxyrhopus species (False Coral Snake). Predation. Herpetological Review, v.48, p.457, 2017.

PINTO, R. R.. Drymoluber dichrous (NCN). Ophiophagy. Herpetol. Rev., v.37, p.231-231, 2006.

SAUCIER, J. R.. Arachnid envenomation. Emergency Medicine Clinics of North America, v.22, n.2, p.405-422, 2004. DOI: https://doi.org/10.1016/j.emc.2004.01.006

SAWAYA, R. J.; MARQUES, O. A. V.; MARTINS, M.. Composição e história natural das serpentes de Cerrado de Itirapina, São Paulo, sudeste do Brasil. Biota Neotrop., v.8, n.2, 2008

SIMON, E.. Etudes arachnologiques. 24e Mémoire. XXXIX. Descriptions d'espèces et de genres nouveaux de la famille des Aviculariidae (suite). Annales de la Société Entomologique de France, v.61, p.271-284, 1892.

VERÍSSIMO, D.; FREITAS, M. M.; ROSA, G. M.. Drymoluber dichrous (northern woodland racer) and Anolis fuscoauratus (slender anole): Predation. Herpetological Bulletin, v.120, p.36-38, 2012.

VIEIRA, W. L. S.; GONÇALVES, M. B. R.; NÓBREGA, R. P.. Predation on Tropidurus hispidus (Squamata: Tropiduridae) by Lasiodora klugi (Aranea: Theraphosidae) in the semiarid caatinga region of northeastern Brazil. Biota Neotropica, v.12, n.4, p.263-265, 2012. DOI: http://dx.doi.org/10.1590/s1676-06032012000400028

WSC. World Spider Catalog. World Spider Catalog. Version 21.0. Natural History Museum Bern, 2020. DOI: https://doi.org/10.24436/2

A CBPC - Companhia Brasileira de Produção Científica (CNPJ: 11.221.422/0001-03) detém os direitos materiais desta publicação. Os direitos referem-se à publicação do trabalho em qualquer parte do mundo, incluindo os direitos às renovações, expansões e disseminações da contribuição, bem como outros direitos subsidiários. Todos os trabalhos publicados eletronicamente poderão posteriormente ser publicados em coletâneas impressas sob coordenação da Sustenere Publishing, da Companhia Brasileira de Produção Científica e seus parceiros autorizados. Os (as) autores (as) preservam os direitos autorais, mas não têm permissão para a publicação da contribuição em outro meio, impresso ou digital, em português ou em tradução. 